\title{
The Effects of Total Sleep Deprivation on Cerebral Responses to Cognitive Performance
}

\author{
Sean P.A. Drummond, Ph.D., and Gregory G. Brown, Ph.D.
}

We review the findings from a study utilizing functional magnetic resonance imaging (FMRI) to examine the effects of total sleep deprivation (TSD) on verbal learning, arithmetic, and divided attention. For verbal learning and divided attention, TSD was associated with increased activation in the bilateral prefrontal cortex and parietal lobes. Increased sleepiness after TSD and lower levels of memory impairment were correlated with increased activation in specific regions of the prefrontal cortex and parietal lobes, respectively. The arithmetic task led to significantly decreased activation in the bilateral prefrontal cortex and parietal lobes. Based on this and other data, we hypothesize an adaptive cerebral response during cognitive performance following TSD with the specific pattern of adaptation depending on the specific cognitive processes performed. We discuss the need to test the hypothesis in a variety of ways.

[Neuropsychopharmacology 26:S68-S73, 2001] (C) 2001 American College of Neuropsychopharmacology. Published by Elsevier Science Inc.
KEY WORDS: Sleep deprivation; Verbal learning; Arithmetic; Divided attention; FMRI; Prefrontal cortex; Parietal lobes

Understanding the impact of sleep deprivation on cognitive performance is increasingly important in a society where many people acutely or chronically fail to obtain adequate sleep. Recent advances in functional brain imaging techniques allow researchers to relate brain function more directly than ever to behavioral performance following sleep deprivation.

Prior to our study described below, only three published neuroimaging studies have concomitantly examined the effects of total sleep deprivation (TSD) on cerebral function and behavioral performance in normal control participants. These studies utilized either positron emission tomography (PET) (Thomas et al. 2000; Wu et al. 1991) or functional magnetic resonance

From the Department of Psychiatry, University of California, San Diego and San Diego Veterans Affairs Medical Center, San Diego, CA. Address correspondence to: Sean P. A. Drummond, Ph.D., Department of Psychiatry, 9116A, University of California, San Diego, San Diego Veterans Affairs Medical Center, 3350 La Jolla Village Dr., San Diego, California 92161, Tel.: (858) 642-1274, Fax: (858) 458-4201, E-mail: drummond@ucsd.edu imaging (FMRI) (Portas et al. 1998) to study brain activation during performance of attention-demanding tasks. Both of the PET studies reported decreased behavioral performance following TSD, decreased global levels of glucose metabolism, and decreased local activation in attention and arousal-related brain regions such as the thalamus. The FMRI study (Portas et al. 1998) utilized a short-lasting visual reaction time task that resulted in equal performance before and after TSD. The authors reported that the thalamus showed an increased hemodynamic response to the attention task only following TSD. Together, these studies suggest that activity levels in brain systems involved in arousal and attention may influence cognitive performance following TSD. The serial addition/subtraction task used by Thomas et al. (Thomas et al. 2000) required arithmetic working memory in addition to the attentional demands and showed decreased activation in related regions such as prefrontal cortex, inferior parietal lobe, and anterior cingulate gyrus. This suggests that brain regions involved in working memory and arithmetic might be vulnerable to TSD.

We recently reported the results from a study utilizing FMRI to examine the effects of $35 \mathrm{~h}$ TSD on cogni- 
tive performance (Drummond et al. 1999, 2000, 2001). The study was aimed at comparing cerebral activation during performance of verbal learning, arithmetic, and divided attention tasks after normal sleep to that following TSD. Table 1 provides a summary of the overall findings. The findings from individual tasks are summarized below, but readers are referred to the primary articles for more details on methodology and results.

\section{Verbal Learning}

This task was designed to highlight brain regions involved in verbal learning and memorization (Drummond et al. 2000). After the normal night of sleep, the verbal learning task activated brain regions typically reported for such tasks (Frackowiak 1994). Following TSD, we did not find the expected general decrease in cerebral activation predicted by the PET studies reviewed above. Rather, each of the regions within left prefrontal cortex showing a significant response after normal sleep also showed a significant response following TSD as did several new regions, including additional prefrontal areas and the bilateral inferior and superior parietal lobes (Figure 1). Following TSD, increased levels of subjective sleepiness were correlated with increased activation in two ventral prefrontal regions. Lower levels of impairment on free recall after TSD (i.e., relative preservation of performance), were correlated with greater activation in the bilateral parietal lobes, including the language areas of the left inferior parietal lobe.

\section{Arithmetic}

The design of this task aimed to activate brain regions involved in arithmetic working memory (Drummond et al. 1999). After normal sleep, we observed the expected activation in bilateral prefrontal and parietal lobe arithmetic working memory regions (Dehaene et al. 1999). Following TSD, however, only the left superior parietal lobe and left premotor area remained sig- nificantly responsive to task demands. Unlike verbal learning, no new areas of activation appeared following TSD. Increased subjective sleepiness following TSD was correlated with activation in one ventral prefrontal region.

Behavioral performance was not correlated with activation in any brain region.

\section{Divided Attention}

The divided attention task combined our verbal learning and arithmetic tasks (Drummond et al. 2001). The cerebral response to divided attention following TSD was very similar to that seen with our verbal learning task. Specifically, several brain regions, particularly within the right prefrontal cortex, bilateral parietal lobes, and cingulate gyrus showed a stronger response to task demands after TSD than after normal sleep. Furthermore, greater levels of subjective sleepiness were significantly correlated with activation in one of the same ventral prefrontal regions seen for the verbal learning task. Finally, as with verbal learning, less impairment in free recall performance following TSD was significantly correlated with activation in the bilateral parietal lobes.

\section{DISCUSSION}

Both the verbal learning and divided attention tasks showed greater cerebral BOLD (blood oxygen level dependent) responses in the bilateral prefrontal cortex and parietal lobes following TSD. We suggest this recruitment of additional brain regions to participate in performance of a cognitive task following TSD represents an adaptive cerebral compensatory response to the detrimental effects of TSD.

\section{Specific Brain Regions May Be Related to Specific Compensatory Functions}

One deficit for which the brain may need to compensate during TSD is the decreased response of the left

Table 1. Summary of TSD Effects on Neuroimaging Data in the Three Cognitive Tasks

\begin{tabular}{lccccccccc}
\hline Task & L. PFC & R. PFC & $\begin{array}{c}\text { L. PMA } \\
\text { \& SMA }\end{array}$ & $\begin{array}{c}\text { ANT. } \\
\text { CING. }\end{array}$ & L. TL & R. TL & L. PL & R. PL & L. Cb \\
\hline Verbal & $\uparrow, S$ & $\uparrow, S$ & $\downarrow, P$ & $=$ & $\varnothing$ & $\downarrow, P$ & $\uparrow, P$ & $\uparrow, P$ & $\varnothing$ \\
$\begin{array}{l}\text { Learning } \\
\text { Arithmetic }\end{array}$ & $\downarrow, \mathrm{S}$ & $\downarrow$ & $\downarrow$ & $\downarrow$ & & & $\downarrow$ & $\downarrow$ & \\
$\begin{array}{l}\text { Divided } \\
\text { Attention }\end{array}$ & $\uparrow, \mathrm{S}$ & $\uparrow$ & $=$ & $\uparrow, \mathrm{S}$ & $\downarrow, \mathrm{S}$ & $=$ & $\uparrow, \mathrm{P}$ & $\uparrow, \mathrm{P}$ & $\downarrow$ \\
\hline
\end{tabular}

$\mathrm{L}=$ left hemisphere; $\mathrm{R} .=$ right hemisphere; $\mathrm{PFC}=$ prefrontal cortex $(\mathrm{BA} 8,9,10,44,45,46,47) ;$ PMA \& SMA = primary and supplementary motor areas (BA6); ANT. CING. = anterior cingulate gyrus (BA24,25,32); TL. = temporal lobe (BA20,21,22,38, medial regions); PL. = parietal lobes (BA7,39,40); $\mathrm{Cb}=$ cerbellum. $\uparrow:$ regions showing a greater response to the cognitive task after TSD relative to the normal night; $\downarrow$ : regions showing less of a response following TSD; =: regions showing equivalent response on both nights; S: reigons significantly correlated with increased sleepiness following TSD; P: regions significantly correlated with lower levels of impairment in free recall performance following TSD. 


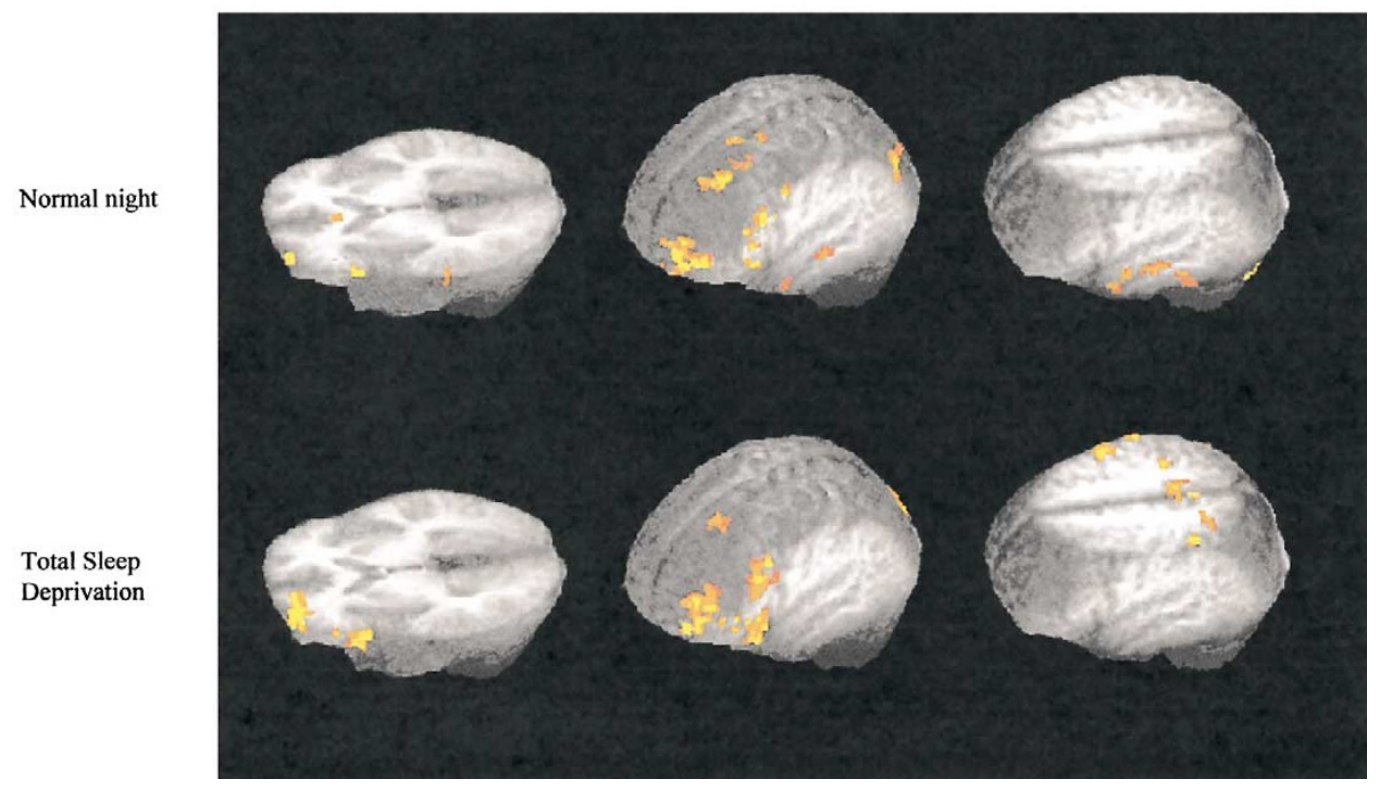

Figure 1. Activation during the verbal learning task. This figure compares brain regions showing a significant response to the verbal learning task after a normal night of sleep (top) and following total sleep deprivation (TSD) (bottom). The first two columns show significant responses on each individual night; the third column shows results of between night analyses. Group activation data is overlayed onto the mean anatomical image from the 13 participants. Colored regions correspond to clusters of significant activation after applying a cluster threshold for Type I error protection; yellow indicates the most intense activation, red the least intense. The left column contains axial slices located $2 \mathrm{~mm}$ above the anterior commisureposterior commisure (AC-PC) line. They show the similar locations of left prefrontal cortex responses (i.e., left orbital and inferior frontal gyri) on each night as well as the increased spatial extent of those responses following TSD. Also shown are anterior cingulate and left middle temporal gyrus on the normal night. The middle column contains sagittal slices located $51 \mathrm{~mm}$ left of the midline. They also show the similar location (left orbital, inferior, and middle frontal gyri) and increased spatial extent of left prefrontal responses. The right column contains two slices: $48 \mathrm{~mm}$ superior to the AC-PC line and 51mm left of the midpoint. The top image shows the greater left temporal activation after normal sleep compared with TSD; the bottom image shows the greater bilateral parietal lobe activation after TSD, as well as right precentral gyrus and precuneus. This last column is adapted from a figure published in Drummond, et al. (2000). Reprinted by permission from Nature 403:655-657, copyright 2000 Macmillan Magazines Ltd.

temporal lobes observed during both verbal learning and divided attention after TSD. This region is typically reported to be involved in a variety of language processing and learning tasks, at least in the normally rested state (Cabeza and Nyberg 2000). The significant correlation between lower levels of impaired memory performance and increased activation in the bilateral parietal lobes following TSD implies the loss of temporal lobe involvement may have been compensated for by bringing the parietal lobes on-line. The involvement of the left inferior parietal lobe in short-term verbal memory storage (Frackowiak 1994; Smith and Jonides 1997) suggests this area as a likely candidate for such a compensatory role. There is also some evidence from research with Alzheimer's Disease (Becker et al. 1996) and Huntington's Disease (Dierks et al. 1998) patients that the parietal lobes can play a compensatory role during cognitive performance. At the same time, this putative "switch" from temporal to parietal involvement may represent a less efficient way of performing these tasks and, thus, may help explain why behavioral performance was not fully intact following TSD.

Compensation for increased sleepiness is also likely necessary following TSD. In all of our tasks, increased levels of sleepiness after TSD were associated with increased BOLD responses in the left ventral prefrontal cortex. Others have found increased EEG activity in frontal regions following sleep deprivation during both waking rest (Cajochen et al. 1999) and cognitive performance (Smulders et al. 1997), and this increased activity, especially that in the theta range, is thought to reflect increased sleepiness. Cajochen et al. (1999) reported that theta EEG power in the frontal derivation was significantly positively correlated with subjective 
sleepiness during TSD. The correlations we observed between increased BOLD signal in the prefrontal cortex and increased sleepiness may reflect the same mechanisms represented by the EEG findings. Each may be a manifestation of neurochemical consequences of prolonged waking and/or increased sleepiness and thus provide a physiological measure of sleepiness. Alternatively, they may reflect a compensatory mechanism designed to combat increased sleepiness, although this remains speculative. Finally, if it is true that the areas of the brain having the greatest activity levels during the day have the "deepest" sleep at night (Horne 1993; Kattler et al. 1994), the correlations we and others have found between sleepiness and increased prefrontal activity of one form or another may reflect the pre-sleep component of this dynamic.

We suggested in this section that increased cerebral responses during TSD may represent a compensatory mechanism. It may be more parsimonious, though, to characterize these findings as an adaptive cerebral response to performance under stress - in this case the stress of TSD. Only in specific circumstances can we argue this adaptation is actually compensating for a deficit. We have outlined two of those cases above and discuss other variables that may relate to adaptation in general, below.

\section{The Adaptive Cerebral Response May Be Related to Cognitive Demands}

While the increased response of the parietal lobes we observed following TSD is consistent with an adaptive (perhaps compensatory) response, neither we nor Thomas et al. (2000) observed a similar pattern during arithmetic tasks of short or long duration, respectively. Therefore, rather than a consistent global response, the cerebral response to cognitive performance following TSD may vary with specific cognitive demands placed on an individual. Our verbal learning and divided attention tasks elicited greater responses after TSD in regions associated with working memory while these regions were not as responsive after a normal night of sleep. On the other hand, these same regions did not show TSD-related greater responsiveness to our arithmetic task but did respond with arithmetic during the normal waking state. This double dissociation suggests the cognitive demands inherent in a task may in part determine the likelihood of an adaptive response after TSD. For example, it may be that since verbal learning tasks typically do not rely heavily on the working memory systems (particularly the executive components thereof), these systems can be tapped after TSD to help compensate for diminished learning resources. Since working memory and arithmetic tasks do normally rely on these same frontal and parietal systems, the resources necessary for compensation may not be available after TSD.
Imaging studies of attention tasks also support the hypothesis that the brain's adaptation to TSD relates to specific task demands. As reviewed above, Portas et al. (1998) reported increased activation after TSD in the thalamus during a short-term attention task, while others (Thomas et al. 2000; Wu et al. 1991) have reported decreased activation in the thalamus after TSD during more long-term attention demanding tasks. Behaviorally, performance on attention tasks declines with increasing time-on-task, particularly after TSD (Dinges and Kribbs 1991). These findings from attention-demanding tasks suggest that cognitive demands may relate not only to the likelihood but also the cerebral location of an adaptive response. The relevant cognitive demands in this set of studies may have been visual attention (relating to the cerebral location of adaptation) and the degree to which attention was sustained over time (relating to the likelihood of adaptation).

\section{Potential Confounds and Future Directions}

If the likelihood and location of cerebral adaptation to sleep deprivation relates to the cognitive demands inherent in a task, why did we see adaptation during verbal learning and divided attention but not during arithmetic? As suggested above, one reason may be the demands placed on the working memory systems by the tasks. One construct often related to working memory is task difficulty. D'Esposito et al. (1995) have reported that increasing task difficulty does not affect prefrontal activation but does increase the extent of parietal lobe activation. Difficulty here, however, is unlikely to explain either the TSD effects on individual tasks or the differences seen among the tasks. Our participants rated the subjective difficulty of each task on each night and these values did not differ between the two nights for any task. Participants consistently rated the verbal learning task the least difficult of the three and the divided attention task the most difficult. The fact we observed cerebral adaptation on the easiest and hardest task suggests difficulty did not directly moderate the observed cerebral responses. Nonetheless, without further research we would not rule out the possibility that working memory load, task difficulty, or some other working memory-related process interacts with task demands to influence the cerebral response following TSD. One way to more systematically test the effects of, for example, task difficulty on the cerebral response is to vary it parametrically within an experiment and identify any brain regions showing a significant relationship with that variation. Our previous task design did not allow for such an analysis.

Another factor that may have influenced our findings is time-on-task. Our cognitive tasks lasted $6 \mathrm{~min}$ each. Would we observe the same type or magnitude of 
increased responses with a substantially longer or shorter task? FMRI is well suited to examining the cerebral response to increasing time-on-task and relating this response to behavioral performance. A third key question is whether the observed adaptive response can be replicated with other types of cognitive tasks. We found adaptation during verbal learning (Drummond et al. 2000) and divided attention (Drummond et al. 2001), and Portas et al. (1998) found it during a visual attention task. Does cerebral adaptation occur during other learning and attention tasks, or during working memory, reasoning, visuo-spatial, or vigilance tasks?

In addition to the various cognitive task-related variables addressed above, a variety of other variables may impact the cerebral response to cognitive performance following TSD. For example, the type and duration of sleep deprivation may affect cerebral responses. Drake et al. (2000) reported that objective measures of sleepiness show an initial decline with partial sleep deprivation and then level off despite continued sleep loss. It is likely that chronic partial and acute total sleep deprivation elicit different types of cerebral responses and characterizing those differences will be important. Circadian factors must also be studied. Our study measured cerebral responses in the early evening when participants were near, if not in, the Wake Maintenance Zone (WMZ). The WMZ is a time of day when the drive of the circadian system is such that sleep is least likely to occur (Strogatz et al. 1987) and cognitive performance is often near its peak (Monk et al. 1997). Thus, it may also be the time of day when cerebral adaptation is most likely to be evident. What would the cerebral response to cognitive performance look like in the early morning hours when the circadian system pulls more for sleep and errors are more likely to occur? In addition to time-of-day effects, variables related to individual differences need examination. Many studies note great variability in participant responses to sleep deprivation, but little is known about what underlies this variability. Do age, sex, or chronotype have any effect on cerebral responses following TSD?

Finally, to fully implement any knowledge we gain from the above types of experiments, we must bring this paradigm to research on populations that typically experience sleep deprivation. Do military personnel, shift workers, or others who experience repeated periods of sleep deprivation continue to show cerebral adaptation or does the strength of such a response diminish? Can the presence or absence of adaptation help us understand the neuropsychological profile of patient populations experiencing sleep deprivation, such as insomnia patients or those with traumatic brain injury? What effects do sleepiness counter measures such as naps, caffeine, and pharmaceuticals have on cerebral responses?

In summary, we hypothesize an adaptive, poten- tially compensatory, cerebral response to cognitive performance during TSD that is related to the cognitive demands placed on an individual. Adaptation is seen as brain regions coming "on-line" and/or showing an increased spatial response to specific cognitive demands following sleep deprivation. The cognitive demands inherent in a given task may help determine both the likelihood and location of this increased cerebral response. Additionally, specific brain regions may be related to specific compensatory functions. Many questions remain before the full nature and extent of this adaptive response can be known. Answers to these questions may ultimately provide insight into the plasticity of the brain and perhaps the function(s) of sleep itself.

\section{ACKNOWLEDGMENTS}

We would like to thank J. Christian Gillin, M.D. for his years of collaboration, mentoring, support, and advice. The data and ideas reported here would have not come to be without his input and assistance. Research supported by a NIMH individual NRSA (SPAD), a Mental Health Clinical Research Center grant from NIMH (J. Christian Gillin, M.D., director), the UCSD General Clinical Research Center (Michael Ziegler, MD, Director), a NIMH institutional NRSA (Michael R. Irwin, M.D., Director), the Department of Veterans Affairs research service, and by the VA Desert-Pacific Healthcare Network Mental Illness Research, Education, and Clinical Center (MIRECC). We would also like to thank Richard B. Buxton, Ph.D., Eric C. Wong, M.D., Ph.D., John L. Stricker, and Elizabeth Wang for invaluable assistance.

\section{REFERENCES}

Becker JT, Mintun MA, Aleva K, Wiseman MB, Nichols T, DeKosky ST (1996): Compensatory reallocation of brain resources supporting verbal episodic memory in Alzheimer's disease. Neurology 46:692-700

Cabeza R, Nyberg L (2000): Imaging cognition II: An empirical review of 275 PET and fMRI studies. J Cogn Neurosci $12: 1-47$

Cajochen C, Khalsa SB, Wyatt JK, Czeisler CA, Dijk DJ (1999): EEG and ocular correlates of circadian melatonin phase and human performance decrements during sleep loss. Am J Physiol 277:R640-R649

Dehaene S, Spelke E, Pinel P, Stanescu R, Tsivkin S (1999): Sources of mathematical thinking: behavioral and brain-imaging evidence. Science 284:970-974

D'Esposito M, Detre JA, Alsop DC, Shin RK, Atlas S, Grossman M (1995): The neural basis of the central executive system of working memory. Nature 378:279-281

Dierks T, Linden DEJ, Hertel A, Gunther T, Lanfermann H, Niesen A, Frolich L, Zanela FE, Hor G, Goebel R, Maurer K (1998): Multimodal imaging of residual function and compensatory resource allocation in cortical atrophy: a case of parietal lobe function in a patient with Huntington's disease. Psychiatry Res 84:27-35 
Dinges DF, Kribbs NB (1991): Performing while sleepy: Effects of experimentally-induced sleepiness. In: Monk TH (ed), Sleep, Sleepiness and Performance. New York, Wiley, pp 97-128

Drake CL, Burduvali E, Bonahoom A, Roehrs T, Roth T (2000): Evidence for an adaptive process associated with cumulative sleep loss. Sleep 23:A72

Drummond SP, Brown GG, Gillin JC, Stricker JL, Wong EC, Buxton RB (2000): Altered brain response to verbal learning following sleep deprivation. Nature 403:655-657

Drummond SPA, Brown GG, Stricker JL, Buxton RB, Wong EC, Gillin JC (1999): Sleep deprivation-induced reduction in cortical functional response to serial subtraction. Neuroreport 10:3745-3748

Drummond SPA, Gillin JC, Brown GG (2001): Increased cerebral response during a divided attention task following sleep deprivation. J Sleep Res. 10:85-92

Frackowiak RS (1994): Functional mapping of verbal memory and language. Trends Neurosci 17:109-115

Horne JA (1993): Human sleep, sleep loss and behaviour. Implications for the prefrontal cortex and psychiatric disorder. Br J Psychiatry 162:413-419

Kattler H, Dijk DJ, Borbely AA (1994): Effect of unilateral somatosensory stimulation prior to sleep on the sleep EEG in human. J Sleep Res 3:159-164

Monk TH, Buysse DJ, Reynoldsi CFI, Berga SL, Jarrett DB,
Begley AE, Kupfer DJ (1997): Circadian rhythms in human performance and mood under constant conditions. J Sleep Res 6:9-18

Portas CM, Rees G, Howseman AM, Josephs O, Turner R, Frith CD (1998): A specific role for the thalamus in mediating the interaction of attention and arousal in humans. J Neurosci 18:8979-8989

Smith EE, Jonides J (1997): Working memory: a view from neuroimaging. Cognit Psychol 33:5-42

Smulders FTY, Kenemans JL, Jonkman LM, Kok A (1997): The effects of sleep loss on task performance and the electroencephalogram in young and elderly subjects. Biol. Psychol. 45:217-239

Strogatz SH, Kronauer RE, Czeisler CA (1987): Circadian pacemaker interferes with sleep onset at specific times each day: Role in insomnia. American J of Physiology 253:R172-R178

Thomas M, Sing H, Belenky G, Holcomb H, Mayberg H, Dannals R, Wagner H, Thorne D, Popp K, Rowland L, Welsh A, Balwinski S, Redmond D (2000): Neural basis of alertness and cognitive performance impairments during sleepiness: I. Effects of 24 hours of sleep deprivation on waking human regional brain activity. J Sleep Res 9:335-352

Wu JC, Gillin JC, Buchsbaum MS, Hershey T, Hazlett E, Sicotte N, Bunney WE Jr (1991): The effect of sleep deprivation on cerebral glucose metabolic rate in normal humans assessed with positron emission tomography. Sleep 14:155-162 